\title{
Tissue eosinophilia: a morphological marker for assessing stromal invasion in squamous neoplasms of the larynx
}

\author{
Bhatta RR ${ }^{1}$, Adhikari RC ${ }^{2}$, Sayami G ${ }^{2}$ \\ ${ }^{1}$ Department of Pathology, BP Koirala Memorial Cancer Hospital, Bharatpur, Nepal \\ ${ }^{2}$ Department of Pathology, Institute of Medicine, Tribhuvan University Teaching Hospital, Kathmandu, Nepal
}

\section{Keywords: \\ Eosinophilia; Larynx; Squamous neoplasms}

\begin{abstract}
Background: The assessment of tumour invasion in squamous neoplasms of the larynx poses a diagnostic challenge, especially in small biopsies that are frequently sectioned tangentially. Eosinophilic infiltration is thought to be an adjunctive criterion in determining tumour invasion. We investigated whether thresholds of eosinophilic infiltration in laryngeal squamous neoplasms would aid in determining the presence of invasion.
\end{abstract}

Materials and Methods: Fifty cases of invasive squamous carcinoma, preinvasive squamous neoplasms and benign squamous neoplasms were evaluated. The number of eosinophils per high power field and per 10 high power fields in the stroma adjacent to the neoplastic epithelium were counted and tabulated. For statistical purposes, the elevated eosinophils were defined and categorized as: focally and moderately elevated (5-9/HPF), focally and markedly elevated (>10/HPF), diffusely and moderately elevated (5$19 / 10 \mathrm{HPF})$, and diffusely and markedly increased ( $>20 / 10 / \mathrm{HPF})$.

Results: Eosinophil counts were elevated focally and/or diffusely more frequently in invasive squamous carcinoma than in noninvasive tumours. The increased eosinophil counts, specifically $>10 / \mathrm{HPF}$ and $>20 / 10 \mathrm{HPF}$, were statistically significantly associated with stromal invasion. Greater than 10/HPF and $>20 / 10 \mathrm{HPF}$ had sensitivity, specificity and positive predictive values of $23 \%, 100 \%, 100 \%$ and $11 \%, 100 \%$ and $100 \%$ respectively. Cytology was able to diagnose 33 out of 36 malignant cases. Of 17 cases which were diagnosed as benign on cytology, 3 cases turned out to be malignant on biopsy. The sensitivity and specificity of touch smear cytology are $91.6 \%$ and $100 \%$ respectively.

Conclusion: The elevated eosinophil count in squamous neoplasms of larynx is a morphologic feature associated with presence of tumour invasion. When the number of infiltrating eosinophils exceeds 10/ HPF and or $>20 / 10 \mathrm{HPF}$ in a laryngeal biopsy with squamous neoplasm, it represents an indicator for the possibility of tumour invasion. Similarly, the presence of eosinophils meeting these thresholds in an excisional specimen should prompt a thorough evaluation for invasiveness, when evidence of invasion is absent or is suspected by conventional criteria in the initial sections.

\section{Correspondence:}

Dr. Ranjan Raj Bhatta, MD

Department of Pathology, BP Koirala Memorial Cancer Hospital,

Bharatpur, Nepal

E-mail: crystaliom@yahoo.com 


\section{INTRODUCTION}

Squamous cell carcinoma is the most common malignant tumour arising in the larynx. ${ }^{1}$ Differentiating between preinvasive squamous neoplasm and invasive squamous neoplasm can be challenging in small biopsy specimens, particularly when the tissue is superficial and fragmented, a prominent inflammatory infiltrate obscures the epithelialstroma interface, there is tangential sectioning of the acanthotic squamous epithelium, or when all these features occur in combination. Even in larger excised specimens, the recognition of superficial invasion may be difficult if the invasive component lacks paradoxical maturation and is composed entirely of basaloid-like cells that infiltrate as round nests rather than irregular cords and small nests in a typical desmoplastic stroma. In difficult cases, the existence of an adjunctive feature associated with invasion would be helpful in determining the presence of any degree of invasion or such a feature should raise the suspicion that the lesion may harbor foci of invasion in the specimen that otherwise lacks conventional diagnostic criteria of invasion., ${ }^{2,3}$

A moderate to marked degree of eosinophilia has been reported to be associated with invasion in cases of squamous neoplasms at different sites of the human body. ${ }^{4}$ Spiegel et al. have reported association of eosinophils with the presence of invasion in squamous neoplasms of the cervix and vulva and have proposed that tissue eosinophilia provided an adjunctive morphologic marker in corroborating the presence of invasion., ${ }^{3,5}$ Alrawi et al. and Said et al. in different studies done on squamous neoplasms of larynx also recorded elevated eosinophils in association with tumour invasion. Both of them concluded that elevated eosinophil count in biopsies of squamous neoplasms of the larynx was a histopathologic marker associated with tumour invasion and tissue eosinophilia was rare or absent in cases of noninvasive squamous neoplasms. ${ }^{2,6}$ Thus, it was hypothesized that the degree of stromal eosinophilia may provide a diagnostic criterion for distinguishing invasive squamous neoplasms from noninvasive neoplasms in the larynx. To test this hypothesis, a systematic study was undertaken to investigate whether thresholds of eosinophils in stroma might serve as a morphological marker for the presence of tumour invasion in cases of squamous neoplasms of the larynx.

\section{MATERIALS AND METHODS}

The study was a hospital-based prospective study conducted in the Department of Pathology, Tribhuvan University Teaching Hospital, Kathmandu, Nepal from August 2009 to June 2011. Cases with prior radiation and / or chemotherapy were excluded. All histology specimens at Tribhuvan University Teaching Hospital were fixed in $10 \%$ formalin. Paraffin blocks of $5 \mu \mathrm{m}$ thickness were cut and sections were stained with conventional hematoxylin and eosin. Cases with any degree of invasion were classified as Squamous

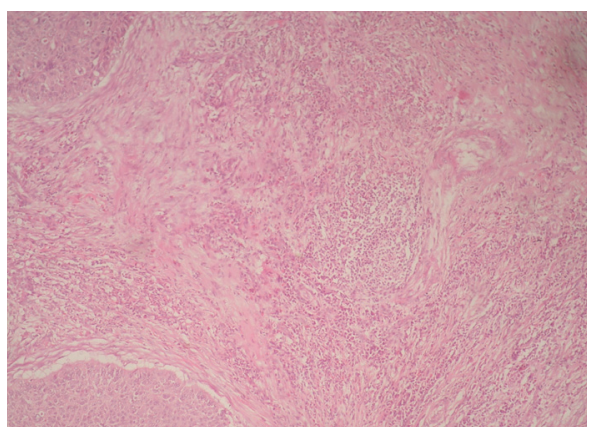

Figure 1: Infiltrative front of the tumour (HE Stain, X100).

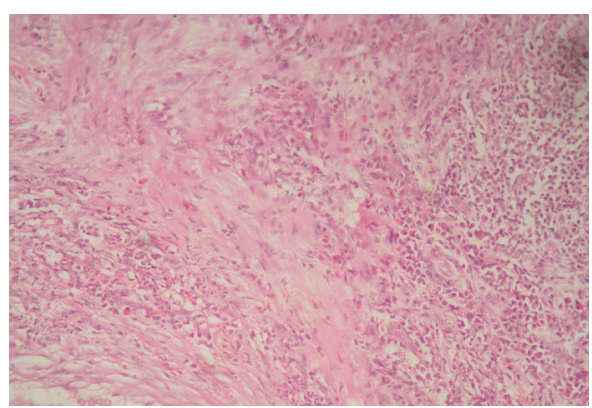

Figure 2: Tumour associated tissue eosinophilia (HE Stain, X400).

cell carcinoma, and graded. Tumours lacking invasion were required for a case to be classified as noninvasive squamous neoplasm. For each specimen, the high power field (Olympus BX51 x10 ocular and x40 objective lens) with maximum number of eosinophils were identified and recorded as eosinophils/hpf (eos/hpf). Subsequently eosinophils in the adjacent nine contiguous high power fields were counted, added to those in the first, and recorded as eosinophils/ 10 high power fields (eos/10hpf). Only nucleated cells with intensely red cytoplasmic granules were accepted as eosinophils and care was taken to exclude red blood cells with superimposed mononuclear and polymorphonuclear inflammatory cells. Eosinophils confined to the lymphovascular spaces were excluded.

Elevated eosinophils were defined and categorized as: focally and moderately elevated (5-9/HPF), focally and markedly increased $(>10 / \mathrm{HPF})$, diffusely and moderately elevated (5-19/10HPF), and diffusely and markedly increased $(>20 / 10 \mathrm{HPF}$ ), while $0-4 / 10 \mathrm{HPF}$ was used as a baseline. The recorded eosinophil counts were analyzed to determine whether there were thresholds of stromal eosinophils per $1 \mathrm{HPF}$ and $10 \mathrm{HPF}$ that were significantly associated with invasive tumor in both biopsy and excisional/resectional specimens.

\section{Statistical methods}

Frequency was computed for each eosinophil category in invasive and noninvasive squamous neoplasia. Modified Fischer test was utilized to examine the difference of frequency distribution between each elevated eosinophil category and the referent eosinophil category $(0-4 / 10 \mathrm{HPF})$. 
Table 1: Distribution of eosinophils in invasive and noninvasive squamous neoplasms

\begin{tabular}{|c|c|c|c|c|c|c|c|c|c|}
\hline \multirow[t]{2}{*}{ Specimen and diagnosis } & \multirow{2}{*}{$\begin{array}{c}\text { Cases\# } \\
0\end{array}$} & \multicolumn{4}{|c|}{ Eosinophils/HPF } & \multirow{2}{*}{$\begin{array}{c}\text { Case\# } \\
0\end{array}$} & \multicolumn{3}{|c|}{ Eosinophils/10HPF } \\
\hline & & $1-4$ & $5-9$ & $10-19$ & $>20$ & & $1-4$ & $5-19$ & $>20$ \\
\hline \multicolumn{10}{|l|}{ Laryngectomy specimen } \\
\hline Invasive Cancer & & 2 & 2 & 3 & 4 & & & 2 & 9 \\
\hline \multicolumn{10}{|l|}{ Noninvasive } \\
\hline \multicolumn{10}{|l|}{ Biopsy specimen } \\
\hline Invasive cancer & 1 & 9 & 3 & 2 & 1 & 1 & 6 & 6 & 3 \\
\hline Noninvasive & 5 & 3 & & & & 5 & 2 & 1 & \\
\hline Benign Lesions & 15 & & & & & 15 & & & \\
\hline
\end{tabular}

Table 2: Significance of eosinophils in invasive and noninvasive squamous neoplasms

\begin{tabular}{lccccccccc}
\hline Specimen and diagnosis & \multicolumn{4}{c}{ Eosinophils/hpf } & \multicolumn{5}{c}{ Eosinophils/10hpf } \\
\hline & $5-9$ & $\mathrm{P}$ & $>10$ & $\mathrm{P}$ & $5-19$ & $\mathrm{P}$ & $>20$ & $\mathrm{P}$ \\
Invasive Cancer & $5 / 27$ & 0.038 & $10 / 27$ & $<0.001$ & $8 / 27$ & 0.022 & $12 / 27$ & $<0.001$ \\
Noninvasive & $0 / 23$ & & $0 / 23$ & & $1 / 23$ & & $0 / 23$ & \\
\hline
\end{tabular}

Table 3: Predective value of eosinophils in assessing stromal invasion in squamous neoplasms of larynx in biopsy specimens

\begin{tabular}{lcccc}
\hline Eosinophils in squamous cell Ca in biopsy specimen & Sensitivity & Specificity & Positive predictive value & Negative predictive \\
\hline$>10$ Eosinophils/hpf & $23 \%$ & $100 \%$ & $100 \%$ & $69 \%$ \\
$>20$ Eosinophils/10hpf & $11 \%$ & $100 \%$ & $100 \%$ & $36 \%$ \\
\hline
\end{tabular}

Sensitivity, specificity and positive predictive value were computed for eosinophil counts that were exceeding $10 /$ HPF or $20 / 10 \mathrm{HPF}$ to evaluate if these two thresholds of eosinophils would suggest any significance of clinical implication from the statistical point of view. P value less than 0.05 was used to determine statistical significance.

\section{RESULTS}

A total of 69 specimens of larynx were received at the Department of Pathology, Tribhuvan University Teaching Hospital during the study period. Eleven were resection specimens and fifty eight were biopsy specimens. Out of the 58 biopsy specimens, 19 were excluded due to lack of adequate stroma. A total of 11 resection specimens and 39 biopsy samples were included in the study.

The distribution of eosinophil counts is summarized in Table 1. Both diffuse eosinophilia ( $>20 / 10 \mathrm{HPF})$ and focal elevated eosinophilic infiltration $(>10 / \mathrm{HPF})$ were noted in invasive tumour in resection specimens (fig.1 and 2). Eosinophilic infiltration was absent in any of the specimen of squamous papilloma. Focal eosinophilic infiltrate was also lacking in the stroma of cases of dysplasia while diffuse moderate eosinophilia (5-19/10HPF) was seen in only one specimen of dysplasia.
As shown in Table 2, 12 cases (44\%) of invasive carcinoma were found to have diffuse eosinophilic infiltration (>20/10HPF); whereas increased eosinophils were not diffusely observed in noninvasive lesions $(p<0.05)$. The same applied for focally marked eosinophilia ( $>10 / \mathrm{HPF})$ when compared with the noninvasive group. Stromal eosinophilia was statistically significantly associated with invasion in squamous cell carcinoma.

In biopsy specimens, diffuse elevation of eosinophils (>20/10HPF) had a sensitivity, specificity and positive predictive value of invasion of $11 \%, 100 \%$ and $100 \%$ respectively. The predictive values of tissue eosinophils in assessing stromal invasion in squamous neoplastic lesions of larynx in biopsy specimens are presented in Table 3. Values below the threshold of $10 / \mathrm{HPF}$ or $20 / 10 \mathrm{HPF}$ had a predictive value of an absence of invasion of $69 \%$ and $36 \%$ respectively. Since laryngectomy was performed only for invasive cases, predictive values could not be calculated for noninvasive cases as control group could not be established.

\section{DISCUSSION}

Pathologists have mostly used histological features like desmoplastic stromal reaction, intra-stromal foreign body reaction to keratin and the presence of small clusters of intra-stromal tumour cells to assess and recognize invasion. 
However, when evaluating a small, poorly oriented or tangentially cut specimens, the pathologist enters a domain replete with uncertainties. The presence of a morphological feature associated with invasion would be effective in determining whether invasion has occurred in equivocal cases.

Tissue eosinophilia has been reported in invasive carcinoma since past hundred years. In the past, different studies have taken place to recognize elevated eosinophil counts in the underlying stroma as a prognostic factor in different neoplasms. A moderate to marked stromal eosinophilia has been observed in cases of invasive squamous neoplasms of the vagina, penis, skin and nasopharynx along with adenocarcinomas of stomach, large intestine and uterus, and also in invasive urothelial carcinoma. Little similarity is noted in all these sites except that they are all at the body surface. $^{7,8}$ Lowe et al have reported tissue eosinophilia ranging from $11.6 \%$ to $20 \%$ in squamous neoplasms at different sites. ${ }^{9}$ Wasserman et al. have described association of tissue eosinophilia with malignant tumour of lungs, especially large cell undifferentiated carcinoma. ${ }^{10}$

Although, different studies have reported association of eosinophilic infiltrate with different cancers, only a few have explored the relationship of such eosinophilic infiltrate with tumour invasion. Spiegel et al. have reported the association of eosinophils with invasion in neoplastic squamous lesions in the female genital tract, and have suggested that eosinophilia provided an adjunctive morphologic feature in identifying squamous cell carcinoma. ${ }^{3,5}$

Said et al in a similar study done on squamous neoplasia of larynx found that elevated eosinophil count in the squamous neoplasms of larynx may serve as a morphologic feature associated with tumour invasion. ${ }^{2}$ Alrawi et al. also remarked that elevated eosinophil counts in biopsies in specimen of the squamous cell carcinoma of the upper aerodigestive tract are a histopathologic marker associated with invasion. ${ }^{6}$ Oliviera et al. have suggested that intense tissue eosinophilia was closely associated with invasion in cases of oral squamous cell carcinoma. ${ }^{11}$

In the current study which consists of a total of 27 cases of invasive carcinoma, focal moderate eosinophilia and focal severe eosinophilia were seen in 5 cases $(18.5 \%)$ and 10 cases $(37 \%)$ respectively. Eight cases $(29.6 \%)$ showed diffuse moderate eosinophilia while diffuse severe eosinophilia was observed in $44.4 \%$ of cases. These findings were statistically significant $(\mathrm{p}<0.05)$ and suggested that elevated eosinophil count could be used as a morphological marker for establishing tumour invasion.

However, association of elevated eosinophils with invasion in biopsy specimens was not statistically significant in this study. This could be alluded to the fact that the small area sampled in the biopsy specimens may not be representative of the invasive front and the whole tumour as opposed to resection specimens.

In a similar study of laryngeal squamous neoplasms with similar morphological criteria, Said et al observed that significant focal and diffuse elevation of eosinophils in invasive squamous carcinomas were statistically more frequent than in noninvasive neoplasia. ${ }^{2}$

In another study done in head and neck squamous neoplasms, Alrawi et al concluded that eosinophilic counts were elevated focally and/or diffusely more frequently in invasive squamous cell carcinoma than in noninvasive tumours and increased eosinophilic counts, specifically $>10 / \mathrm{HPF}$ and $>20 / 10 \mathrm{HPF}$, were both statistically associated with stromal invasion. ${ }^{6}$

Current study demonstrated that $>10 / \mathrm{HPF}$ and $>20 / 10 \mathrm{HPF}$ had a sensitivity of $23 \%$ and $11 \%$ respectively, which was relatively low in comparison to the sensitivity of $82 \%$ and $80 \%$ in the study by Said et al. and $66 \%$ and $61 \%$ found by Alrawi et al. The specificity and positive predictive value of elevated eosinophil counts in biopsy specimens was high, similar to the findings of Said et al and Alrawi et al. ${ }^{2,6}$

Although elevated eosinophil counts are statistically significantly associated with stromal invasion in squamous cell carcinoma of larynx, occasionally the presence of high number of eosinophils were observed in noninvasive specimens (Table 1). Thus, the presence of eosinophils in squamous neoplasia of larynx is not pathognomonic for stromal invasion and caution must be exercised when evaluating the number of infiltrating eosinophils. However, the quantification method and thresholds identified in the current experiment may represent an adjunctive feature in the assessment of stromal invasion in squamous neoplasia. Specifically, the presence of eosinophils at these thresholds should raise the suspicion that invasive or microinvasive carcinoma is present within the specimen, particularly when $>10 / \mathrm{HPF}$ and/or 20/10HPFs are observed.

\section{CONCLUSION}

The current study suggests that an elevated eosinophil count in laryngeal squamous neoplasms is a feature of invasion. The presence of more than individual eosinophils, when it exceeds the threshold limits, $>10 / \mathrm{HPF}$ and or $>20 / 10 \mathrm{HPF}$, in a biopsy of larynx with squamous neoplasm, represents a morphological marker for the presence of invasion and provides supportive evidence for invasion in cases with equivocal invasion. Such elevated counts should raise the suspicion that a lesion may harbor foci of invasion when it is absent by conventional diagnostic criteria and warrant additional sections and/or levels to search for invasion. 


\section{REFERENCES}

1. Pilch BZ. Larynx and trachea. In: Fletcher CDM, editor. Diagnostic histopathology of tumours. 3rd ed.Elsevier Ltd: Philadelphia; 2007. pp150.

2. Said M, Wiseman S, Yang J et al. Tissue eosinophilia: a morphologic marker for assessing stromal invasion in laryngeal squamous neoplasms. BMC Clin Pathol 2005;5:1-8.

3. Spiegel GW. Eosinophils as a marker for invasion in vulvar squamous neoplastic lesions. Int J Gynecol Pathol 2002;21:108-16.

4. Alkhabuli JO, High A. Significance of eosinophil counting in tumour associated tissue eosinophilia. Oral Oncol 2006;42:849-50.

5. Spiegel GW, Ashraf M, Brooks JJS. Eosinophils as a marker for invasion in cervical neoplastic lesions. Int J Gynecol Pathol $2002 ; 21: 117-24$
6. Alrawi SJ, Dongfeng T, Stoleer DL et al. Tissue eosinophilic infiltration: a useful marker for assessing stromal invasion, survival and locoregional recurrence in head and neck squamous neoplasia. Cancer J 2005;11:217-25.

7. Dorta RG, Landman G, Kowalski LP, Lauris JR, Latorre MR, Oliviera DT. Tumour- associated tissue eosinophilia as a prognostic factor in oral squamous cell carcinoma. Histopathology 2002;41:152-7.

8. Samoszuk M. Eosinophils and human cancer. Histol Histopathol 1997; 12:807-12.

9. Lowe D, Fletcher CD. Eosinophilia in squamous cell carcinoma of the oral cavity, external genitalia and anus- clinical correlations. Histopathology 1984;8:627-32.

10. Wasserman SI, Goetzl EJ, Ellman L, Austen KF. Tumour associated eosinophilotactic factor. N Engl J Med 1974;290:420-4.

11. Oliviera DT, Tjioe KC, Assao A et al. Tissue eosinophilia and its association with tumoral invasion of oral cancer. Int J Surg Pathol $2009 ; 17: 244-9$ 